\begin{tabular}{|c|c|}
\hline Title & Polarization anisotropic luminescence of tunable single lateral quantum dot molecules \\
\hline Author(s) & Hermannstädter, C.; Witzany, M.; Heldmaier, M.; Hafenbrak, R.; Jöns, K. D.; Beirne, G. J.; Michler, P. \\
\hline Citation & $\begin{array}{l}\text { Journal of A pplied Physics, 111(6), } 063526 \\
\text { https://doi.org/10.1063/1.3697982 }\end{array}$ \\
\hline Issue Date & 2012-03-15 \\
\hline Doc URL & http:/hdl.handle.net/2115/49210 \\
\hline Rights & $\begin{array}{l}\text { Copyright } 2012 \text { A merican Institute of Physics. This article may be downloaded for personal use only. A ny other use } \\
\text { requires prior permission of the author and the A merican Institute of Physics. The following article appeared in J. A ppl. } \\
\text { Phys. 111, } 063526 \text { (2012) and may be found at https://dx.doi.org/10.1063/1.3697982 }\end{array}$ \\
\hline Type & article \\
\hline File Information & JA P111-6_063526.pdf \\
\hline
\end{tabular}

Instructions for use 


\section{AIP Applited Physics}

\section{Polarization anisotropic luminescence of tunable single lateral quantum dot molecules}

C. Hermannstädter, M. Witzany, M. Heldmaier, R. Hafenbrak, K. D. Jöns et al.

Citation: J. Appl. Phys. 111, 063526 (2012); doi: 10.1063/1.3697982

View online: http://dx.doi.org/10.1063/1.3697982

View Table of Contents: http://jap.aip.org/resource/1/JAPIAU/v111/i6

Published by the American Institute of Physics.

\section{Related Articles}

Capping effect of $\mathrm{GaAsSb}$ and InGaAsSb on the structural and optical properties of type II GaSb/GaAs quantum dots

Appl. Phys. Lett. 100, 171914 (2012)

Identification of visible emission from $\mathrm{ZnO}$ quantum dots: Excitation-dependence and size-dependence

J. Appl. Phys. 111, 083521 (2012)

Chromium-oxide enhancement of photo-oxidation of CdSe/ZnS quantum dot solids

J. Appl. Phys. 111, 084308 (2012)

Internal structure of tunable ternary CdSexS1-x quantum dots unraveled by $\mathrm{x}$-ray absorption spectroscopy

Appl. Phys. Lett. 100, 163113 (2012)

Formation of coupled three-dimensional GeSi quantum dot crystals

Appl. Phys. Lett. 100, 153113 (2012)

\section{Additional information on J. Appl. Phys.}

Journal Homepage: http://jap.aip.org/

Journal Information: http://jap.aip.org/about/about_the_journal

Top downloads: http://jap.aip.org/features/most_downloaded

Information for Authors: http://jap.aip.org/authors

\section{ADVERTISEMENT}

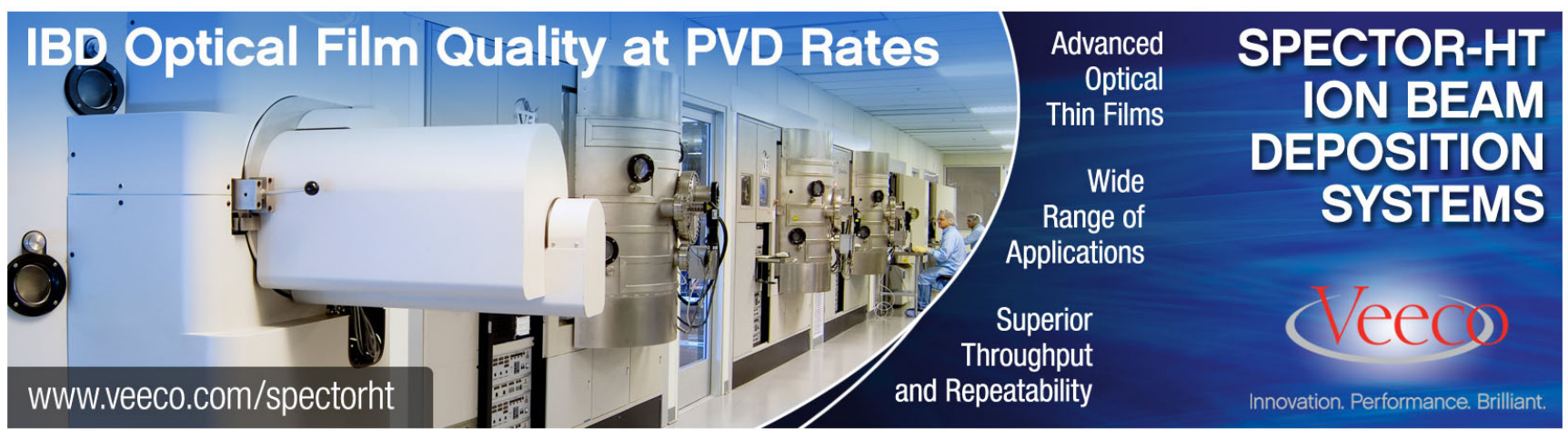




\title{
Polarization anisotropic luminescence of tunable single lateral quantum dot molecules
}

\author{
C. Hermannstädter, ${ }^{1,2, a)}$ M. Witzany, ${ }^{1}$ M. Heldmaier, ${ }^{1}$ R. Hafenbrak, ${ }^{1}$ K. D. Jöns, ${ }^{1}$ \\ G. J. Beirne, ${ }^{1, b)}$ and P. Michler ${ }^{1}$ \\ ${ }^{1}$ Institut für Halbleiteroptik und Funktionelle Grenzfächen, Universität Stuttgart, Allmandring 3, \\ Stuttgart 70569, Germany \\ ${ }^{2}$ Research Institute for Electronic Science, Hokkaido University, Sapporo 001-0021, Japan
}

(Received 28 November 2011; accepted 21 February 2012; published online 29 March 2012)

\begin{abstract}
We investigate the photoluminescence polarization anisotropy of self-assembled individual lateral InGaAs/GaAs quantum dot molecules. In contrast to similarly grown single quantum dots, the dot molecules exhibit a remarkable degree of linear polarization, which remains almost unchanged when a lateral electric field is applied to tune the exciton wave function and, thus, the luminescence spectral properties. We discuss the nature of this polarization anisotropy and suggest possible causes based on the system's symmetry and heterostructure alloy composition. () 2012 American Institute of Physics. [http://dx.doi.org/10.1063/1.3697982]
\end{abstract}

\section{INTRODUCTION}

In quantum information technology, solid state nanostructures have become key components to generate "flying" messenger photon qubits and to realize reliable photon sources for entanglement-based quantum cryptography. Selfassembled semiconductor quantum dots (QDs) stand out as promising basic units for such applications, e.g., as sources for triggered single photons as well as entangled photon pairs. ${ }^{1-5}$ Based on these individual QDs, larger and more functional units can be designed and realized by vertically stacking them along the growth direction ${ }^{6-10}$ and/or laterally arranging them. ${ }^{1-14}$ These more complex units can be used not only as single photon and entangled photon pair sources for the aforementioned applications, but also to act as basic quantum gates on charges and spins that are confined within them. ${ }^{15,16}$

The polarization properties of QDs that are used as entangled photon pair sources, such as the exciton fine structure (FS) and its tuning, are very important and have been widely discussed, both for single InGaAs/GaAs QDs ${ }^{17-20}$ and QD molecules (QDMs). ${ }^{21,22}$ Tuning the FS splitting (FSS) of the neutral exciton state to values on the order of its natural linewidth is a promising approach to use the respective radiative biexciton-exciton cascaded emission process to produce polarization entangled photon pairs. ${ }^{3-5}$ Strain-induced anisotropic exchange interaction (AEI) and valence-band mixing (VBM) might have an impact on the exciton FS, even for highly symmetric single QDs and QDMs, and are recently also discussed as possible sources for polarization anisotropy. ${ }^{23,24}$ Especially the symmetry of QD structures and their confinement potentials, as well as their impact on the polarization of the related excitonic recombination, are important topics in current theoretical and experimental studies..$^{25,26}$

\footnotetext{
a)Electronic mail: claus@es.hokudai.ac.jp.

b) Present address: Huygens Laboratory, Leiden University, P.O. Box 9504, Leiden 2300 RA, Netherlands.
}

In this report, we investigate the polarization properties of single photons emitted from lateral InGaAs/GaAs QDMs manipulated by an electric field. In particular, we analyze and discuss the high degree of linear polarization (DLP) of these molecules in comparison to similar single dots, which were grown in a comparable way and which exhibit similar recombination energies. We suggest possible reasons for the observed polarization anisotropy of the investigated QDMs and discuss their consequences and impact on the theoretical description and their future application in quantum information processing.

\section{THE DOT MOLECULE SAMPLES}

The low density $\left(1 \times 10^{8} \mathrm{~cm}^{-2}\right)$ QDMs were grown on $\mathrm{GaAs}(001)$ substrates by molecular beam epitaxy and are consistently aligned along the $[1 \overline{1} 0]$ crystal direction. A "basin", which was formed in the GaAs barrier by in situ etching, was subsequently filled with $\operatorname{In}_{y} \mathrm{Ga}_{1-y} \mathrm{As}$ and followed by the growth of the two $\operatorname{In}_{x} \mathrm{Ga}_{1-x}$ As QDs at the edges of the basin. The QDs have heights of approximately $2.8 \mathrm{~nm}$, widths of $40-48 \mathrm{~nm}$, and an edge-to-edge distance of $4-11 \mathrm{~nm} .{ }^{12-14}$ The gradients in the In content of dots and ba$\sin , x$ and $y$, are most significant along the growth direction (Fig. 1(a)). A more detailed discussion of the structural data and a theoretical model are presented in Refs. 14 and 17, respectively. The total QDM active layer is embedded inside a planar lambda-cavity with 20 bottom pairs and one top pair of AlAs/AlGaAs distributed Bragg reflectors in order to obtain a higher photoluminescence (PL) extraction at the typical emission energy of around $1.35 \mathrm{eV}^{22}$ The reliable and repeatable alignment of all QDMs along the [11 10$]$ direction allows for the application of electric fields along a controlled direction with respect to the QDM coupling axis. The static electric fields are created by voltage control of parallel metal electrodes that are deposited on top of the sample with spacings of 5 to $20 \mu \mathrm{m}$ between them.

Detailed atomistic empirical pseudopotential calculations of the same kind of lateral QDMs over a basin were 


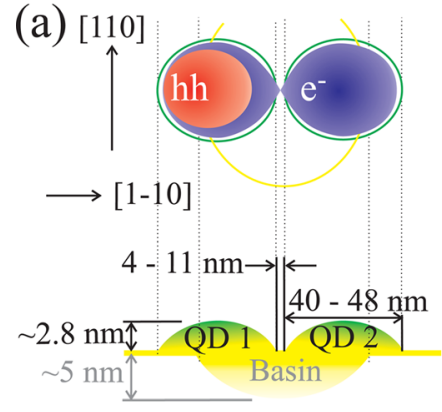

In content $50 \% \square 0 \%$

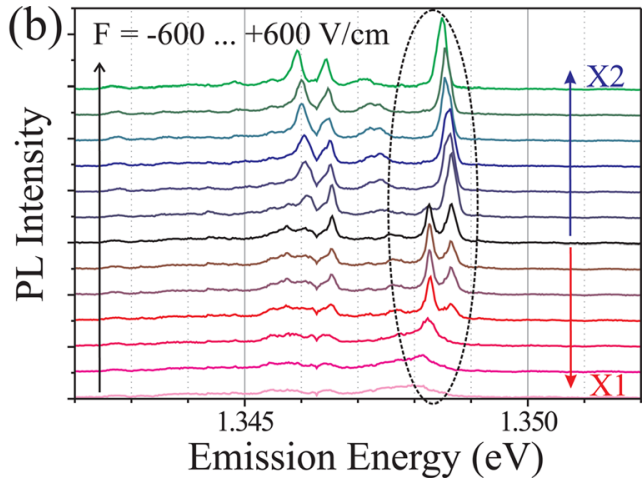

FIG. 1. (a) Schematic top view of a lateral QDM with the two QDs over the basin and a possible combination of single-particle electron ( ${ }^{-}$) and heavy hole (hh) wave functions; cross-sectional view of the QDM illustrating its alignment, dimensions, and the In gradient. (b) Electric field tuning of the excitonic emission from lateral $Q D M A$ under non-resonant cw excitation below saturation power $\left(200 \mathrm{Wcm}^{-2}\right)$. The spectra are obtained for $F=-600$ to $+600 \mathrm{~V} / \mathrm{cm}$ with an increment of $100 \mathrm{~V} / \mathrm{cm}$ and are displayed with artificial vertical offsets for clarity. The dashed ellipse highlights the change in the relative intensities of the two neutral exciton transitions, X1 and X2. The spectral features at lower energies are due to biexcitonic recombination and are not further discussed here.

carried out and are reported in Refs. 27 and 28. Therein, the calculated electronic level structure of single particles, neutral and charged excitons, as well as multi-excitons are presented. Furthermore, the calculated wave function dependence on a static lateral electric field is discussed and is in line with the observed changes to the QDM PL spectra. In that theoretical description, as well as in previous experimental studies, we have highlighted that electron tunnel coupling is possible, even at the rather large center-to-center distance between the two dots. As a consequence, the ground state electron single particle wave function or state density, $\langle e \mid e\rangle$, in the QDM can spread over both dots, as schematically sketched in Fig. 1(a), and can furthermore be tuned using the lateral electric field along the coupling axis. By tuning this electric field, the electron state density can be shifted from one dot via an equal distribution over both dots (at $F=F_{e}$ ) to the other dot. The hole, on the other hand, due to its larger effective mass, experiences the QDM confinement potential as two rather separated dots. Its ground state density, $\langle h \mid h\rangle$, thus remains almost completely localized in either of the dots (Fig. 1(a)) and shows no significant tunnel coupling. ${ }^{13,27}$

\section{OPTICAL SPECTROSCOPY}

\section{A. Low-temperature micro-photoluminescence}

Low-temperature micro-PL measurements were carried out in a helium flow cryostat at $5 \mathrm{~K}$ by using an objective lens with $\mathrm{NA}=0.45$, which results in a focus diameter of about $1.5 \mu \mathrm{m}$. The low spatial QDM density directly allows for the examination of one QDM at a time without any further sample structuring, such as mesa etching or shadow mask fabrication. The optical excitation was performed using either a cw or a 3-ps pulsed Ti:sapphire laser at $800 \mathrm{~nm}$ $(1.55 \mathrm{eV})$, which photo-generates carriers in the bulk GaAs barrier and thus non-resonantly creates excitons of no distinct spin-configuration (polarization) in the QDM. The excitation power was kept below or close to the neutral exciton saturation level, such that a high PL signal and reasonably low background could be obtained. The QDM PL was col- lected with the same objective lens, dispersed by a $75-\mathrm{cm}$ spectrometer with a $12001 / \mathrm{mm}$ grating and detected using a silicon charge coupled device camera.

The electrical tuning of the QDM charge distribution and, consequently, of the optical emission properties was performed via a lateral electric field. The field was applied along the QDM coupling axis, [1 10$]$, through parallel titanium-gold electrodes, as described above. The PL spectra for electric fields between -600 and $+600 \mathrm{~V} / \mathrm{cm}$ are displayed in Fig. 1(b). The field-dependence clearly reveals the switching of the relative intensities of the two peaks, $\mathrm{X} 1$ and $\mathrm{X} 2$, which is highlighted by the dashed ellipse. We attribute the origin of these recombination lines to neutral exciton recombination, with the hole in either of the two dots that make up the QDM. For example, the low-energy peak X1 corresponds to the recombination in QD 1 according to the configuration illustrated in Fig. 1(a). A weak (close to) quadratic Stark shift can be seen featuring the maximum exciton recombination energies for $F \approx 100 \mathrm{~V} / \mathrm{cm}$. This type of Stark shift is characteristic of lateral QDM excitons and is indicative of a negligible intrinsic electric dipole moment and a rather small polarizability. In other words, the wave function overlap integral, $\langle e \mid h\rangle$, tends to be rather stable and unaffected by lateral electric fields up to a few $\mathrm{kV} / \mathrm{cm}$, which is furthermore supported by almost unchanged exciton radiative lifetimes on the order of $0.7-1 \mathrm{~ns}^{29,30}$

The corresponding second-order (intensity) autocorrelation measurements under ps-pulsed excitation (Fig. 2) highlight the triggered single-photon nature of the electric field tunable neutral exciton recombination, X1 and X2. For all three tuning situations, with $\mathrm{X} 1$ dominant, $\mathrm{X} 1$ and $\mathrm{X} 2$ equally intense at $F_{e}$, and $\mathrm{X} 2$ dominant, the antibunching value, $g^{2}(\tau=0)$, is below 0.3 , which confirms the single photon nature of the QDM emission. The related crosscorrelations between different QDM PL lines are presented elsewhere. ${ }^{13,22,29}$

\section{B. Polarization analysis of the dot molecule Iuminescence}

One major interest when studying the polarization properties of QDs is their FS. This FS is believed to be a key 


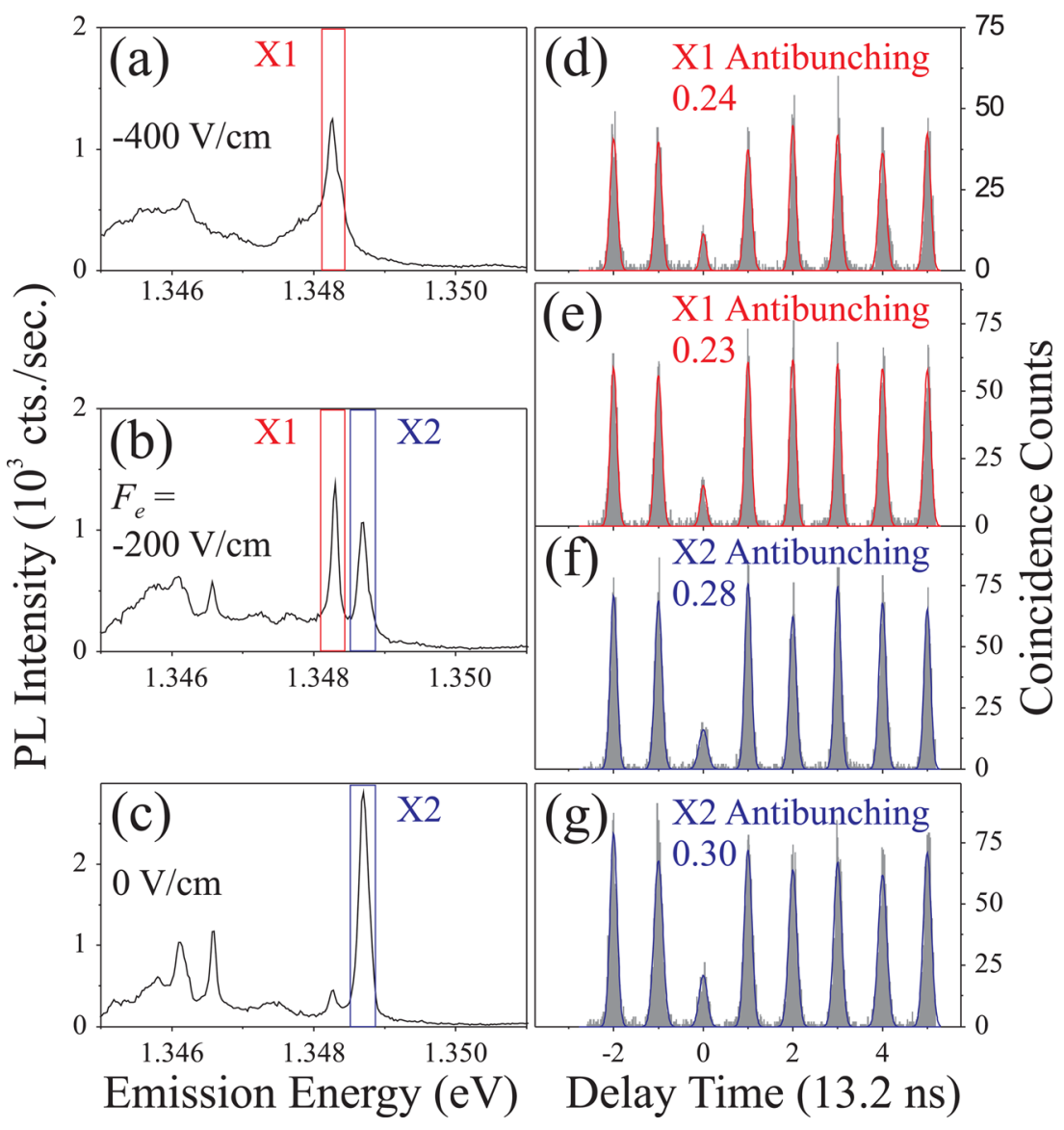

FIG. 2. The curves on the left display the PL spectra of $Q D M A$ obtained under ps-pulsed non-resonant excitation close to saturation power $\left(500 \mathrm{Wcm}^{-2}\right)$ for three different tuning situations: (a) X1 dominant at $F=-400 \mathrm{~V} / \mathrm{cm}$, (b) $\mathrm{X} 1$ and $\mathrm{X} 2$ tuned to similar intensities at $F=F_{e}=-200 \mathrm{~V} / \mathrm{cm}$, and (c) $\mathrm{X} 2$ dominant at $F=0 \mathrm{~V} / \mathrm{cm}$. The graphs on the right (d)-(g) display the corresponding second-order autocorrelation histograms for these tuning situations. All evaluated antibunching values are obtained directly from the histograms without background subtraction, post-measurement modification, or deconvolution of the instrument response function. aspect for the possibility of generating polarization entangled photon pairs from QDs by using a cascaded emission process. While the first comprehensive studies on radiative cascades in vertical QDMs were reported in Ref. 21, we previously reported on the biexciton-exciton cascades in lateral QDMs. ${ }^{22}$ In that work, a FSS on the order of $10 \mu \mathrm{eV}$ was measured and two uncharged radiative cascades could be identified. However, the polarization anisotropy, i.e., the polarization degree of the QDM PL has not been investigated until now and is discussed below.

The polarization isotropy or anisotropy of the emitted luminescence is of particular interest when coupled QD systems, such as lateral QDMs, are investigated. Obviously, the symmetry of the lateral QDM, treated as one entity, is not only lower than the ideal assumption of a single QD or vertical QDM of perfectly cylindrical $\left(D_{2 d}\right)$ symmetry, but is also lower than the more realistic $C_{2 v}$ or $C_{3 v}$ symmetry that appears in these systems as a result of the substrate orientation, e.g., (001) or (111). ${ }^{26}$

We investigate the PL polarization as a function of applied lateral electric field. Therefore, the QDMs were nonresonantly excited without any modulation of the excitation laser polarization. The PL signal, in turn, was analyzed with respect to its polarization properties by rotating a half-wave plate (HWP) placed in front of a fixed linear polarizer (LP). The PL intensities, which are displayed in Figs. 3(d)-3(g), were obtained by fitting peaks X1 and X2 in PL spectra that were recorded at various HWP angles. The results obtained at three distinct QDM neutral exciton tuning situations are featured, namely, the case of a dominant X1 exciton peak, two equally intense excitons, $\mathrm{X} 1$ and $\mathrm{X} 2$, at $F_{e}$, and the $\mathrm{X} 2$ exciton as the dominant peak. The PL intensity maxima, $I_{\text {max }}$, are consistently found at polarization angles close to zero and $\pm 180^{\circ}$, which corresponds to the direction along the QDM coupling axis $[1 \overline{1} 0]$. The PL intensity minima, $I_{m i n}$, are found perpendicular to that axis at polarization angles of around $\pm 90^{\circ}$. We defined the polarization angle as twice the HWP rotation angle with respect to the LP axis and set the zero point by using the linear polarization of the laser together with the sample orientation. The linear polarization degree is

$$
\mathrm{DLP}=\frac{I_{\text {max }}-I_{\text {min }}}{I_{\text {max }}+I_{\text {min }}} .
$$

At this point, we would like to recall and stress the single photon nature of the individual PL peaks, X1 and X2, as demonstrated in Fig. 2, in order to rule out the possibility that two uncorrelated features of different polarization overlap accidentally in the PL spectra. We also want to highlight that the FSS of the presented QDM was below the resolution limit of our detection system, including line-shape fitting, i.e., the FSS $\leq 10 \mu \mathrm{eV}$.

The projections of the PL of the neutral excitons of about a dozen of other QDMs have been investigated in the same way; the intensity maxima were consistently found at directions parallel to the QDM coupling axis with deviations smaller than $\pm 10^{\circ}$ and with comparably small FSS. Anisotropic linear polarization could be observed for every investigated neutral exciton in lateral QDMs with DLPs on the 


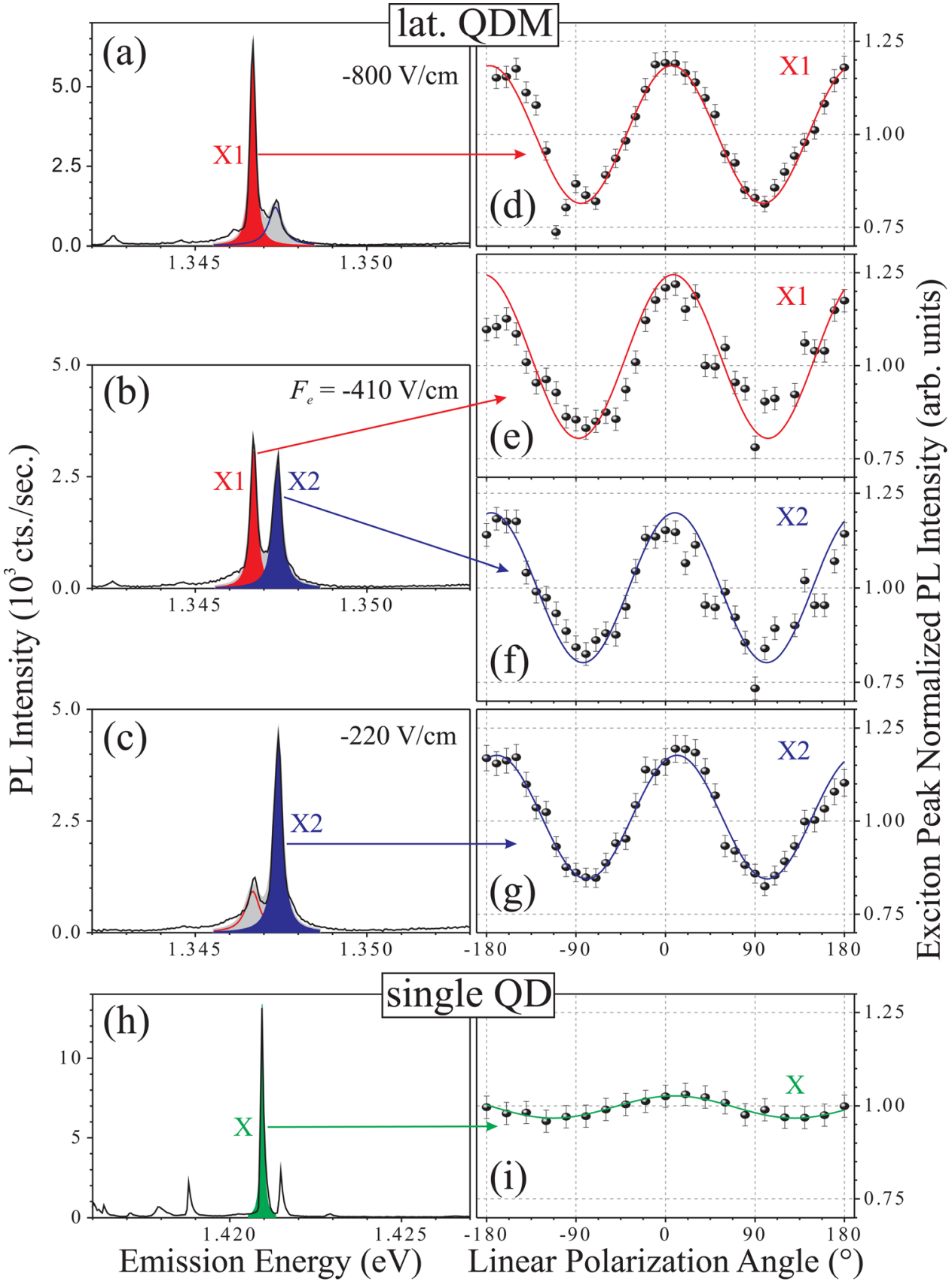

FIG. 3. $Q D M B$ PL spectra (non-resonant excitation at $300 \mathrm{Wcm}^{-2}$ ) are displayed on the left for the three different tuning situations and at a polarization angle of zero degrees: (a) X1 dominant at $F=-800 \mathrm{~V} / \mathrm{cm}$, (b) X1 and X2 tuned to similar intensity at $F=F_{e}=-410 \mathrm{~V} / \mathrm{cm}$, and (c) X2 dominant at $F=-220 \mathrm{~V} / \mathrm{cm}$. The corresponding linear polarization plots are displayed on the right, where the linear polarization axis of 0 and $\pm 180^{\circ}$ correspond to the $[110]$ crystal axis. The fitted polarization degrees are (d) DLP(X1) $=18 \pm 1 \%$, (e) DLP(X1) $=19 \pm 2 \%$, (f) DLP(X2) $=18 \pm 2 \%$, and (g) DLP(X2) $=16 \pm 1 \%$. The single QD data are displayed for comparison: (h) the PL spectrum and (i) the linear polarization plot, showing a comparatively low $\mathrm{DLP}_{\mathrm{QD}}(\mathrm{X}) \sim 3 \%$ of the neutral exciton $\mathrm{X}$.

order of 5-30\% and an average of around $12.5 \%$; however, no significant and clear influence of the applied electric field on the DLP could be found. The change in DLP when varying the electric field is either zero or within/close to the uncertainty of the measurement results (typically $\sim 2 \%$ ). This means that the case of both excitons appearing equally intense at $F_{e}$, which might be associated with a slightly smaller wave function overlap, $\langle e \mid h\rangle$, shows no significant difference in polarization to the case where both the electron and the hole are localized within the same dot (larger $\langle e \mid h\rangle$ ). Nevertheless, we want to point out that, within the measure- ment uncertainty, the DLP at $F_{e}$ shows a tendency to be slightly larger (up to 2-3\%).

A comparison between single InGaAs/GaAs dots and lateral QDMs can be done based on the results shown in Figs. 3(d) $-3(\mathrm{~g})$ and 3(i). The QDMs exihibit a mean DLP of approximately $12.5 \%$, whereas the typical single dot DLP is, on average, $3.6 \%$. From several dozens of measured dots, we selected data from a single QD exciton that exhibits a detectable polarization degree, which is clearly not perfectly aligned along one of the crystal (symmetry) axes. The majority of QDs do not show any significant polarization 
anisotropy; a value of $7.6 \%$ is the maximum DLP found for all investigated dots so far. More importantly, even in the case where there exists an observable polarization anisotropy, no distinct and reproducible directionality is observed, and the angle between the polarization components can differ from $90^{\circ}$, which is in good agreement with recent theoretical considerations and experimental reports. ${ }^{24,31,32}$

AEI and VBM could well explain the observed small polarization of the single QD PL under the assumption of a much weaker electron-light hole transition compared to the electron-heavy hole transition. However, these contributions alone cannot explain the generally higher polarization of the lateral QDM PL. If the latter processes were the only contributing effects or mechanisms, the wave function tuning and, thus, the change in VBM should influence the DLP much more significantly.

In the following paragraph, we want to take a look at our QD structures from a more detailed microscopic viewpoint. In ternary materials, such as in (In,Ga)As, the cations (In and $\mathrm{Ga}$ atoms) are usually assumed to be distributed at random positions in the crystal. In the idealized case of a

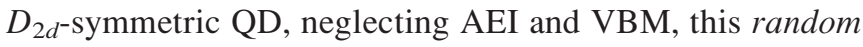
alloy should lead to unpolarized PL, i.e., to a crystal without microscopic local optical dipoles. In a more realistic approach, however, as theoretically discussed by Mlinar and Zunger in Ref. 32, it might instead lead to an observable "effect of atomic-scale randomness on the optical polarization". The described randomness in the simulated problem is attributed to the limited number of atoms in a QD $\left(\leq 10^{5}\right)$, which can result in a random and significant FS and polarization of different orientation and magnitude $(\leq 5 \%)$. Their theoretical results are in good agreement with our single dot experimental results and may be the reason for the small and randomly oriented polarization. In the lateral QDMs, in stark contrast, a fixed and reproducible polarization axis is observed. This observation can therefore be most likely attributed to the appearance of aligned microscopic optical dipoles due to (partial) local ordering of In and Ga atoms within the QDMs and/or due to a specific intrinsic geometry and alignment of the strain and piezoelectric field.

Our observation and description of significantly different DLPs for single QDs and lateral QDMs of similar growth, dimensions, and overall composition leads to the necessity of a modified and extended theoretical description of QDMs when optical polarization effects are to be considered. Very recently, this effect of local ordering of In and Ga atoms was also theoretically found to lead to a DLP of up to $25 \%$ for single InGaAs QDs. ${ }^{33}$ This corresponds well to the findings discussed in this article. The aforementioned considerations of microscopic optical dipoles and alloy ordering, however, do not completely apply to the discussion related to the FS and FS tuning. This is because the FS is mainly determined by the system's symmetry, the extension of the confinement potential, and the wave function overlap and tuning, i.e., by the macroscopic electric dipole and the respective electron-hole exchange interactions. That means, even if the lateral QDM PL appears to be polarized with a DLP of around 20\%, its polarization FSS can still be very small $(\mathrm{FSS} \leq 10 \mu \mathrm{eV})$ and remain the subject of efforts to tune it to sufficiently small values to use the QDM as a source of entangled photon pairs.

\section{SUMMARY}

In summary, we investigated the linear polarization anisotropy of single photons emitted from tunable lateral InGaAs/GaAs QDMs. The QDM excitons consistently show an anisotropy in linear polarization of $12.5 \%$ on average, which is attributed to microscopic optical dipoles that may be the result of local ordering of In and Ga atoms in the ternary alloy. In contrast to similarly grown single QDs, which show only small polarization anisotropy of $3.6 \%$ on average with no preferred orientation, the lateral QDMs exhibit a significantly higher DLP and a reproducible polarization orientation along the QDM coupling axis. Nevertheless, their intrinsic exciton FSS is smaller than $10 \mu \mathrm{eV}$. We believe this experimental report highlights the future importance of a more detailed understanding of the microscopic atomic-scale structure of non-binary alloy QD systems when polarization phenomena are described, both from the experimental and theoretical point of view.

\section{ACKNOWLEDGMENTS}

The authors thank L. Wang, A. Rastelli, M. Wiesner, W.-M. Schulz, M. Eichfelder, R. Rossbach, M. Jetter, and M. Ubl for their contributions to the sample growth and processing, as well as J. Peng, G. Bester, H. Sasakura, and I. Suemune for valuable discussions. Work at the University of Stuttgart was financially supported by the Deutsche Forschungsgemeinschaft via SFB/TRR 21 and FOR 730. C.H. acknowledges the Japan Society for the Promotion of Science (JSPS) for providing financial support in the form of a JSPS Fellowship for Foreign Researchers.

${ }^{1}$ Single Semiconductor Quantum Dots, NanoScience and Technology, edited by P. Michler (Springer, Berlin, 2009).

${ }^{2}$ P. Michler, A. Kiraz, C. Becher, W. V. Schoenfeld, P. M. Petroff, L. Zhang, E. Hu, and A. Imamoglu, Science 290, 2282 (2000).

${ }^{3}$ R. M. Stevenson, R. J. Young, P. Atkinson, K. Cooper, D. A. Ritchie, and A. J. Shields, Nature 439, 179 (2006).

${ }^{4}$ N. Akopian, N. H. Lindner, E. Poem, Y. Berlatzky, J. Avron, D. Gershoni, B. D. Gerardot, and P. M. Petroff, Phys. Rev. Lett. 96, 130501 (2006).

${ }^{5}$ R. Hafenbrak, S. M. Ulrich, P. Michler, L. Wang, A. Rastelli, and O. G. Schmidt, New J. Phys. 9, 315 (2007).

${ }^{6}$ G. S. Solomon, J. A. Trezza, A. F. Marshall, and J. S. Harris, Jr., Phys. Rev. Lett. 76, 952 (1996).

${ }^{7}$ M. Bayer, P. Hawrylak, K. Hinzer, S. Fafard, M. Korkusinski, Z. R. Wasilewski, O. Stern, and A. Forchel, Science 291, 451 (2001).

${ }^{8}$ H. J. Krenner, M. Sabathil, E. C. Clark, A. Kress, D. Schuh, M. Bichler, G. Abstreiter, and J. J. Finley, Phys. Rev. Lett. 94, 057402 (2005).

${ }^{9}$ G. Ortner, M. Bayer, Y. Lyanda-Geller, T. L. Reinecke, A. Kress, J. P. Reithmaier, and A. Forchel, Phys. Rev. Lett. 94, 157401 (2005).

${ }^{10}$ E. A. Stinaff, M. Scheibner, A. S. Bracker, I. V. Ponomarev, V. L. Korenev, M. E. Ware, M. F. Doty, T. L. Reinecke, and D. Gammon, Science 311, 636 (2006).

${ }^{11}$ O. G. Schmidt, C. Deneke, S. Kiravittaya, R. Songmuang, H. Heidemeyer, Y. Nakamura, R. Zapf-Gottwick, C. Müller, and N. Y. Jin-Phillipp, IEEE J. Sel. Top. Quantum Electron. 8, 1025 (2002).

${ }^{12}$ R. Songmuang, S. Kiravittaya, and O. G. Schmidt, Appl. Phys. Lett. 82, 2892 (2003). 
${ }^{13}$ G. J. Beirne, C. Hermannstädter, L. Wang, A. Rastelli, O. G. Schmidt, and P. Michler, Phys. Rev. Lett. 96, 137401 (2006).

${ }^{14}$ L. Wang, A. Rastelli, S. Kiravittaya, P. Atkinson, F. Ding, C. C. Bof Bufon, C. Hermannstädter, M. Witzany, G. J. Beirne, P. Michler, and O. G. Schmidt, New J. Phys. 10, 045010 (2008).

${ }^{15}$ L. Robledo, J. Elzerman, G. Jundt, M. Atatüre, A. Högele, S. Fält, and A. Imamoglu, Science 320, 772 (2008).

${ }^{16}$ D. Kim, S. G. Carter, A. Greilich, A. S. Bracker, and D. Gammon, Nature Phys. 7, 223 (2011)

${ }^{17}$ K. Kowalik, O. Krebs, A. Lemaître, S. Laurent, P. Senellart, P. Voisin, and J. A. Gaj, Appl. Phys. Lett. 86, 041907 (2005).

${ }^{18}$ B. D. Gerardot, S. Seidl, P. A. Dalgarno, R. J. Warburton, D. Granados, J. M. Garcia, K. Kowalik, O. Krebs, K. Karrai, A. Badolato, and P. M. Petroff, Appl. Phys. Lett. 90, 041101 (2007).

${ }^{19}$ M. M. Vogel, S. M. Ulrich, R. Hafenbrak, P. Michler, L. Wang, A. Rastelli, and O. G. Schmidt, Appl. Phys. Lett. 91, 051904 (2007).

${ }^{20}$ R. Singh and G. Bester, Phys. Rev. Lett. 104, 196803 (2010).

${ }^{21}$ M. Scheibner, M. F. Doty, I. V. Ponomarev, A. S. Bracker, E. A. Stinaff, V. L. Korenev, T. L. Reinecke, and D. Gammon, Phys. Rev. B 75, 245318 (2007).

${ }^{22}$ C. Hermannstädter, M. Witzany, G. J. Beirne, W.-M. Schulz, M. Eichfelder, R. Rossbach, M. Jetter, P. Michler, L. Wang, A. Rastelli, and O. G. Schmidt, J. Appl. Phys. 105, 122408 (2009).
${ }^{23}$ C.-H. Lin, W.-T. You, H.-Y. Chou, S.-J. Cheng, S.-D. Lin, and W.-H. Chang, Phys. Rev. B 83, 075317 (2011).

${ }^{24}$ S. Ohno, S. Adachi, R. Kaji, A. Muto, and H. Sasakura, Appl. Phys. Lett. 98, 161912 (2011)

${ }^{25}$ M. A. Dupertuis, K. F. Karlsson, D.Y. Oberli, E. Pelucchi, A. Rudra, P. O. Holtz, and E. Kapon, Phys. Rev. Lett. 107, 127403 (2011).

${ }^{26}$ A. Schliwa, M. Winkelnkemper, A. Lochmann, E. Stock, and D. Bimberg, Phys. Rev. B 80, 161307 (2009).

${ }^{27}$ J. Peng, C. Hermannstädter, M. Witzany, M. Heldmaier, L. Wang, S. Kiravittaya, A. Rastelli, O. G. Schmidt, P. Michler, and G. Bester, Phys. Rev. B 81, 205315 (2010).

${ }^{28}$ J. Peng and G. Bester, Phys. Rev. B 82, 235314 (2010).

${ }^{29} \mathrm{C}$. Hermannstädter, Optical properties of single charge-tunable and laterally coupled (In,Ga)As/GaAs quantum dots, Ph.D. dissertation, Verlag Dr. Hut, München, 2010).

${ }^{30}$ C. Hermannstädter, G. J. Beirne, M. Witzany, M. Heldmaier, J. Peng, G. Bester, L. Wang, A. Rastelli, O. G. Schmidt, and P. Michler, Phys. Rev. B 82, 085309 (2010).

${ }^{31}$ G. Bester, private communication (2011).

${ }^{32}$ V. Mlinar and A. Zunger, Phys. Rev. B 79, 115416 (2009).

${ }^{33}$ R. Singh and G. Bester, Phys. Rev. B 84, 241402 (2011). 